\title{
Correction to: An end-to-end differential network learning method for semantic segmentation
}

\author{
Tai Hu${ }^{1}$ (D) Ming Yang ${ }^{1} \cdot$ Wanqi Yang ${ }^{1} \cdot$ Aishi Li $^{1}$
}

Published online: 8 January 2019

○) Springer-Verlag GmbH Germany, part of Springer Nature 2019

\section{Correction to: \\ International Journal of Machine Learning and Cybernetics \\ https://doi.org/10.1007/s13042-018-0889-3}

Unfortunately, the Fig. 8 and the acknowledgment section was published incorrectly in the online published article. The correct figure and acknowledgment are given below. The original article has been corrected.

The original article can be found online at https://doi.org/10.1007/ s13042-018-0889-3.

\section{Tai Hu}

hutaizn@foxmail.com

$\triangle$ Ming Yang

myang@njnu.edu.cn

1 School of Computer Science and Technology, Nanjing

Normal University, Nanjing 210023, China

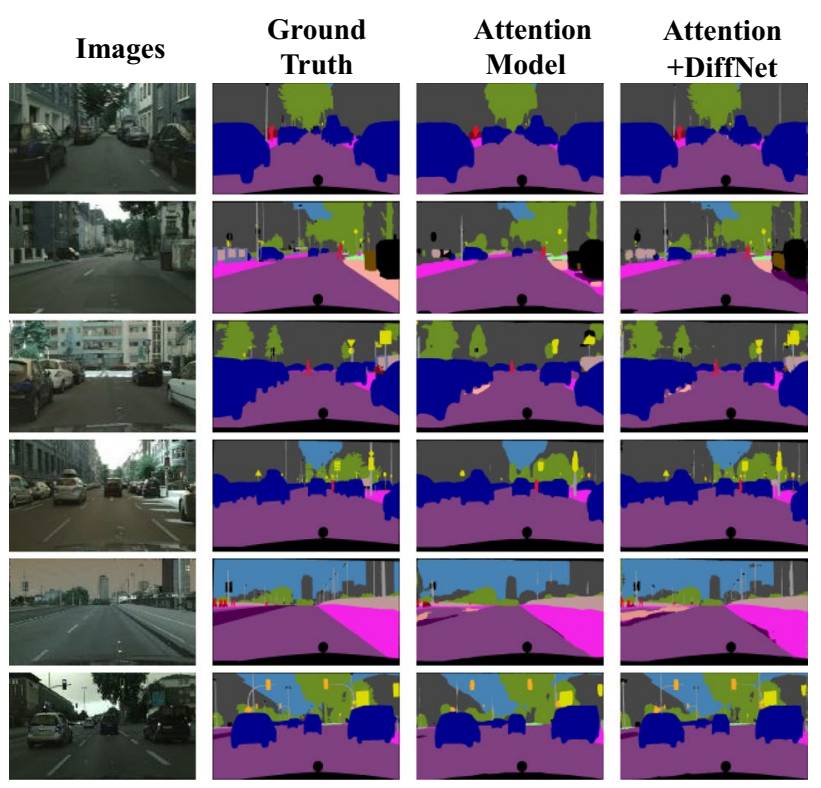

Fig. 8 Qualitative visual comparisons on Cityscapes new test set between attention model and Attention+DiffNet.

Acknowledgements This work is supported by National Natural Science Foundation of China (61876087, 61432008, 61272222, 61603193), Natural Science Foundation of Jiangsu Province (BK20171479, BK20161020, BK20161560), and Program of Natural Science Research of Jiangsu Higher Education Institutions (15KJB520023).

Publisher's Note Springer Nature remains neutral with regard to jurisdictional claims in published maps and institutional affiliations. 